Research Article

\title{
Extreme-Point Symmetric Mode Decomposition to Define the Turbulence Characteristics of a Flume Flow
}

\author{
Zhi-Peng Shi $\mathbb{D}^{1}{ }^{1}$ Ting-Ting He, ${ }^{1}$ and Gen-Guang Zhang ${ }^{2}$ \\ ${ }^{1}$ Jiangsu Vocational Institute of Architectural Technology, Xuzhou, Jiangsu 221116, China \\ ${ }^{2}$ College of Water Resources and Architectural Engineering, Northwest A \& F University, Yangling, Shaanxi712100, China
}

Correspondence should be addressed to Zhi-Peng Shi; szp821002@126.com

Received 1 February 2021; Accepted 29 June 2021; Published 9 July 2021

Academic Editor: Adnan Maqsood

Copyright (C) 2021 Zhi-Peng Shi et al. This is an open access article distributed under the Creative Commons Attribution License, which permits unrestricted use, distribution, and reproduction in any medium, provided the original work is properly cited.

Turbulence is a key feature of solid-liquid two-phase flows, and the pulsating velocity is the basis for calculating turbulence characteristics. In general, the method of mathematical expectation is used to calculate pulsating velocity. However, this method does not reflect the fluctuating state of the instantaneous velocity. Therefore, the method of extreme-point symmetric mode decomposition (ESMD) is adopted to calculate pulsating velocity and turbulence characteristics. The ESMD involves two stages, namely, modal decomposition and time-frequency analysis. The optimal adaptive global mean (AGM), which is the result of modal decomposition, can accurately reflect the fluctuation state of the instantaneous velocity, and the theory of the pulsating velocity defined on this basis is reasonable. Moreover, the flow pattern and turbulence behaviour of a two-phase flow can be predicted using the calculated turbulence characteristics. The method is used to analyse the pulsating velocity of the flume, and its rationality in theoretically predicting the turbulence behaviour of flume flows is demonstrated.

\section{Introduction}

The suspension of solid particles in turbulent flows can be widely observed in nature, such as in the case of fine sediments in fluvial and estuarine environments [1-3]. Owing to the frequent occurrence of such flows, the complex phenomena pertaining to the interaction of solid particles and turbulence must be examined. Many experimental and computational studies related to solid particles in openchannel turbulent flows have been reported, for example, studies pertaining to sediment transportation and the measurement of the pulsating velocity of liquid-solid twophase flows [4-7].

At present, the use of dynamic response signals to analyse turbulence is a key research topic pertaining to twophase flows. In this context, the turbulence characteristics must be tracked based on the acquired nonstationary velocity response signals. In particular, due to the time-varying process of the flume flow, the properties for the turbulence characteristics must be tracked based on nonstationary response signals acquired through acoustic Doppler velocimetry (ADV). Pulsating velocity is a key to identify turbulence characteristics; however, data measured using ADV pertain to the instantaneous velocity. In general, the mathematical mean is usually applied to determine the pulsating velocity [8]. This process of data analysis is known as time-frequency analysis (TFA), which can be conducted using fast Fourier transform (FFT) or wavelet transform (WT). However, FFT is applicable only for linear stationary signals, and prespecified basis functions are required to apply WT. Consequently, FFT and WT cannot be applied to effectively process nonstationary and nonlinear signals [9]. Therefore, an extreme-point symmetric mode decomposition (ESMD) method was proposed to solve the above problems through an optimal global mean curve in an adaptive way and determine the instantaneous frequency and amplitude in a direct way [10]. The ESMD was mostly used in climate research, such as the spatiotemporal variations of nonlinear trends of precipitation [11] or the temporal and spatial patterns of drought in the Yellow River [12]. Ren et al. [13] studied the atmospheric turbulence structure and intermittency during heavy haze pollution in the Beijing area with the method of Hilbert-Huang transform. The ESMD is an improvement of Hilbert-Huang 
transform. This study was aimed at using the ESMD to extract the properties of nonstationary and transient signals. Subsequently, the turbulence characteristics of a flume flow were analysed based on ESMD.

\section{Model Description and Mathematical Method}

2.1. Experimental Model. A flume made of plexiglass with a length, width, and height of $232 \mathrm{~cm}, 16 \mathrm{~cm}$, and $15 \mathrm{~cm}$, respectively, was used. To ensure that the flume flow exhibited a steady state, the ratio of the flume width to its height was approximately 1 , and the height of the sections between sections 2 and 12 was $26.5 \mathrm{~cm}$ (Figure 1) [14]. The flume was divided into 13 sections. The region between sections 2 and 12 was the key research area, and the distance between these two sections was $15 \mathrm{~cm}$. Sand $\left(d_{50}=39.63 \mu \mathrm{m}\right)$ with a concentration of $1 \mathrm{~kg} / \mathrm{m}^{3}$ was applied. The grid and gate valve switch were used to realize a fully developed turbulent flow and stable liquid-solid two-phase flow. Acoustic Doppler velocimetry (Vector, Nortek China Ltd., China) was performed at a sample frequency of $25 \mathrm{~Hz}$ to test the flow velocity.

2.2. Extreme-Point Symmetric Mode Decomposition (ESMD). ESMD is an innovative algorithm based on the Hilbert-Huang transform (HHT). ESMD involves two parts: modal decomposition, in which several intrinsic model functions (IMFs) and an optimal adaptive global mean (AGM) can be generated, and time-frequency analysis pertains to the total energy conversion $[9,10,12]$.

2.2.1. Algorithm of Modal Decomposition. The purpose of modal decomposition is to yield a series of intrinsic model functions (IMFs) and an optimal adaptive mean curve. The process flow is as follows:

Step 1: find all the extreme points of the input data $Y$ and record the points as $E_{i}(i=1,2, \ldots, n)$ sequentially

Step 2: connect the adjacent points with lines and record the midpoints of the lines as $F_{i}(i=1,2$, $\ldots, n-1)$

Step 3: add the left and right boundary midpoints $F_{0}$ and $F_{n}$, respectively.

Step 4: construct $p$ interpolation lines $L_{1}, \ldots, L_{p}(p \geq 1)$ using the $n+1$ midpoints obtained previously and calculate the mean curves as $L^{*}=\left(L_{1}+\cdots+L_{p}\right) / p$

Step 5: if $\left|L^{*}\right| \leq \varepsilon$ or the number of sifting times reaches the maximum value $K$, the first empirical mode $M_{1}$ is decomposed. If not, the abovementioned steps are repeated.

Step 6: repeat the abovementioned steps on the residual $Y-M_{1}$ and obtain the other modes $M_{2}, M_{3}, \ldots$ until the final value $R$ with only a certain number of extreme points is obtained

Step 7: ensure that $K$ is within the integer interval $\left[K_{\min }, K_{\max }\right]$ and that a series of decomposition modes are obtained through the previous steps. Subsequently, calculate the variance $\sigma / \sigma_{0}$ and plot its curve with $K$, where $\sigma$ and $\sigma_{0}$ denote the relative standard deviation of $Y-R$ and the standard deviation of the original data, respectively.

Step 8: find the maximum number of sifting times corresponding to the minimum variance ratio $\sigma / \sigma_{0}$ in the interval $\left[K_{\min }, K_{\max }\right]$. Subsequently, repeat the abovementioned steps to output the decomposition results. The last residual $R$ is the optimal adaptive mean curve.

2.2.2. Algorithm of Instantaneous Frequency. When applying FFT and WT, integral operations must be performed on the data, and the original discrete signals must be converted into analytical functions for processing; however, this processing is limited in terms of mathematical theory. The direct interpolation algorithm uses the average frequency over the local period as the interpolation point to generate a smooth curve. It is assumed that the adjustment of the frequency between the adjacent local periods is gradual. This assumption is consistent with the basic methods of data analysis. The direct interpolation algorithm is used to perform time-frequency analysis. The process flow is as follows.

If the discrete form of the intrinsic model functions (IMFs) is $\left(t_{k}, y_{k}\right), k=1,2, \ldots, N$, the algorithm to determine the instantaneous frequency involves the following steps:

Step 1: determine all of the quasiextreme points of each intrinsic model function that satisfies the following conditions (1).

$$
\begin{aligned}
& y_{k-1}<y_{k} \geq y_{k+1}, \\
& y_{k-1} \leq y_{k}>y_{k+1}, \\
& y_{k-1}>y_{k} \leq y_{k+1}, \\
& y_{k-1} \geq y_{k}<y_{k+1} .
\end{aligned}
$$

Subsequently, enumerate all the points as in set $E=\left(t_{e i}, y_{e i}\right), i=1,2, \ldots, m$

Step 2: define the interpolation frequency points, considering $E=\left(t_{e i}, y_{e i}\right), i=1,2, \ldots, m$

Step 3: add the boundary points by using linear interpolation. For the left boundary, if $y_{1}=y_{e_{1}}$, $a_{1}=t_{1}, f_{1}=0$. Otherwise, use linear interpolation to define the boundary. Identify the right boundary in the same manner as the left boundary.

Step 4: perform cubic spline interpolation to obtain the interpolation curve by using the $m$ time-frequency points. The instantaneous frequency curve can be defined as

$$
f^{*}(t)=\max \{0, f(t)\}
$$

\section{Results and Discussion}

3.1. Signal Acquisition and Analysis. In the experiments, 13 sections were defined in the flume model, as shown in Figure 1. A total of 13 points corresponding to all the sections 


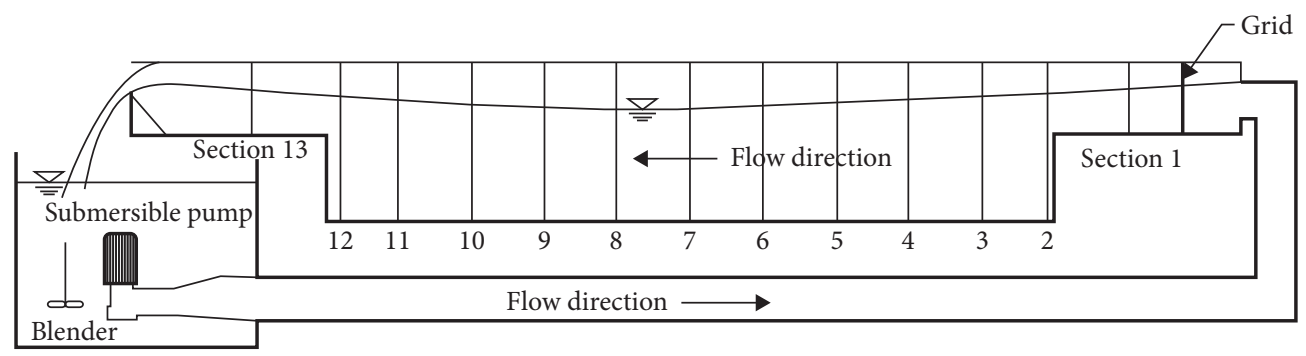

FIGURE 1: Schematic of the experiment setup.

(sections 2-12) from the water surface to the bed were identified, and the instantaneous velocity on the 143 points was examined through the acoustic Doppler velocimetry technique. In addition, an independent coordinate system was established, whose $X, Y$, and $Z$ directions were parallel to the flow direction, parallel to the water depth direction, and perpendicular to the flume width, respectively. The $X, Y$, and $Z$ directions corresponded to the $u, v$, and $w$ velocity components, respectively. The velocities at a distance of $3 \mathrm{~cm}$ from the riverbed at sections 3,7 , and 11 were considered as the data samples for the analysis. Figure 2 shows the instantaneous velocity sample for the considered location.

Figure 2 shows that the instantaneous velocities fluctuate significantly although they exhibit certain regularity in the flow and water depth directions, as shown in Figures 2(d), 2(e), 2(g), and $2(\mathrm{~h})$. The regularity of the instantaneous velocity at section 7 was the highest; the corresponding regularity at section 11 was low and that at section 3 was the lowest. These phenomena can be attributed to the flow pattern of the section. At section 3, similar to jet flows, a strong turbulence occurs, and the flow has a large amount of kinetic energy; therefore, the instantaneous velocity exhibits a higher randomness (Figures 2(a)-2(c)). Section 7 lies in the middle of the flume, and its velocity is the most representative of the main flume flow characteristics. Although section 11 lies at the end of the main flume, the instantaneous velocity of the section still exhibits certain regularity. This regularity, similar to the Sin function, indicates that the liquid-solid two-phase flow exhibits a high fusion and uniformity. In addition, along the flow direction, the value of the instantaneous velocity gradually decreases (Figures 2(a), $2(\mathrm{~d})$, and $2(\mathrm{~g}))$, which is consistent with the flow characteristics of an actual open-channel flow.

3.2. Optimal Adaptive Global Mean. As described in Section 3.1, the flume exhibits typical pulsating characteristics. An actual open channel is considerably influenced by the pulsating characteristics, such as the vibration and ability to carry sediments. The instantaneous velocity consists of two parts: the mean velocity and pulsating velocity. The pulsating velocity is a key-pulsating characteristic and reflects the difference between the instantaneous and mean velocities. The conventional approach to calculate the mean velocity corresponds to the mathematical expectation, which is defined as method 1 herein $[8,15,16]$. If the instantaneous velocity is $V=\left\{v_{i}\right\}_{i=1}^{N}$, the mean velocity can be obtained using equation (3) (method 1).

$$
\bar{V}=\frac{1}{N} \sum_{i=1}^{N} v_{i}
$$

where $\bar{V}$ is the mean velocity, $v_{i}$ is the instantaneous velocity, and $N$ is the number of points for which the instantaneous velocity is considered.

Although method 1 represents the simplest method to calculate the mean velocity, it is not the optimal method. Specifically, method 1 cannot reflect the variation trend of the instantaneous velocity over time. The method involving the use of the optimal adaptive global mean is defined as method 2. Method 2 is a part of the ESMD technique and corresponds to the result of modal decomposition. The specific algorithm is described in Section 2.2. Figure 3 shows the mean velocities calculated using methods 1 and 2 .

As shown in Figure 3, the mean velocities calculated using methods 1 and 2 are considerably different. The mean velocity calculated using method 1 is a fixed value and does not reflect the fluctuations in the instantaneous velocity. However, the mean velocity calculated using method 2 can accurately reflect the fluctuation in the instantaneous velocity. Moreover, the fluctuation law of the mean velocity obtained using method 2 is similar to the law of the Sin function curve, as shown in Figures 3(a), 3(b), 3(d), and 3(e). This law is consistent with that of the instantaneous velocity described in Section 3.1. Therefore, the optimal adaptive global mean velocity (method 2) can more accurately reflect the instantaneous velocity, regardless of the direction and value. The pulsating velocity can be defined in a more precise manner under a more accurate mean velocity.

3.3. Pulsating Velocity. Figure 4 compares the pulsating velocities calculated using methods 1 and 2 . The pulsating velocity calculated using method 1 is equal to the difference between the instantaneous velocity and the mathematical expectation of the velocity. In contrast, the pulsating velocity calculated using method 2 is equal to the difference between the instantaneous velocity and the optimal adaptive global mean velocity. Figures 4(a)-4(y) show the comparison of the pulsating velocities obtained using the two different methods over the entire time period. Figures 4(b), 4(c), 4(e), 4(f), 4(h), 4(i), 4(k), 4(l), 4(n), 4(o), 4(q), 4(r), 4(t), 4(u), 4(w), $4(\mathrm{x}), 4(\mathrm{z})$, and $4(\mathrm{a} 1)$ show the segments of the maximum difference in the pulsating velocity corresponding to Figures 4(a)-4(y). 


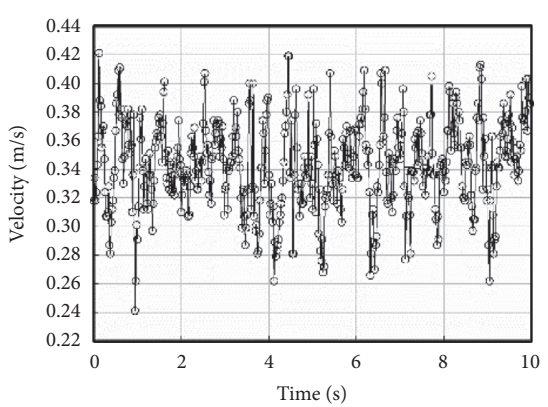

(a)

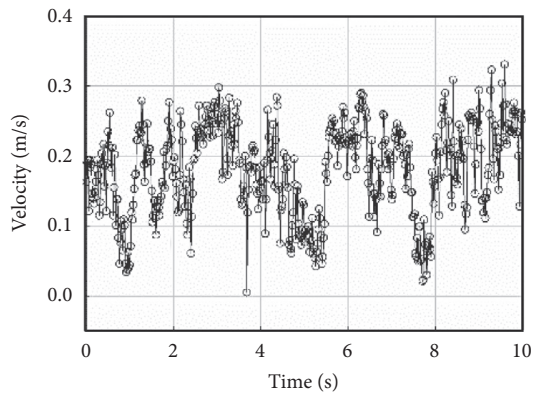

(d)

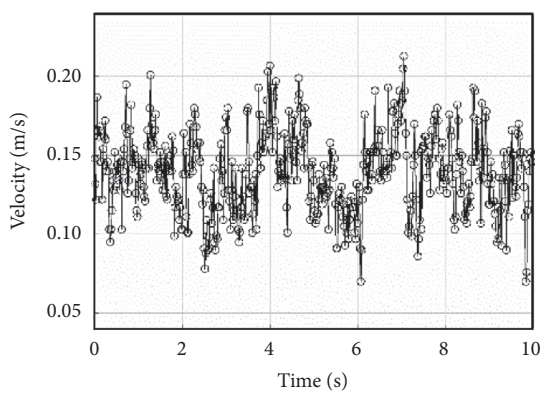

(g)

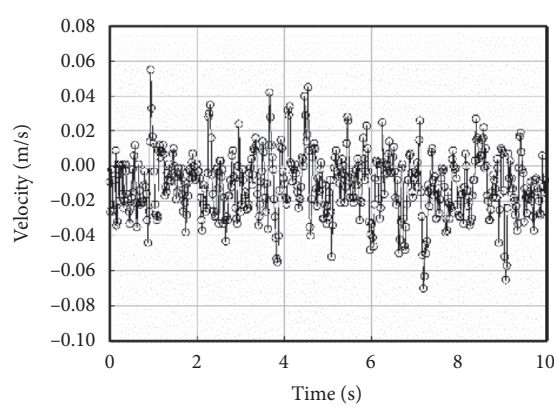

(b)

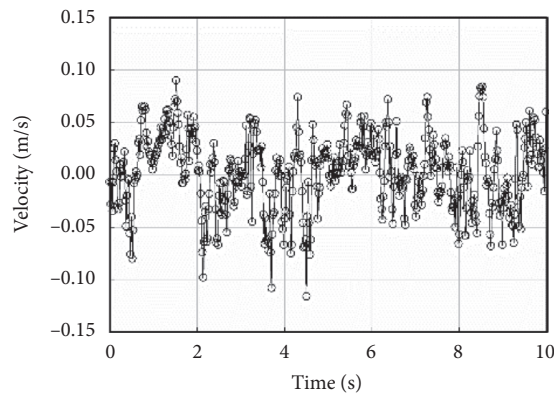

(e)

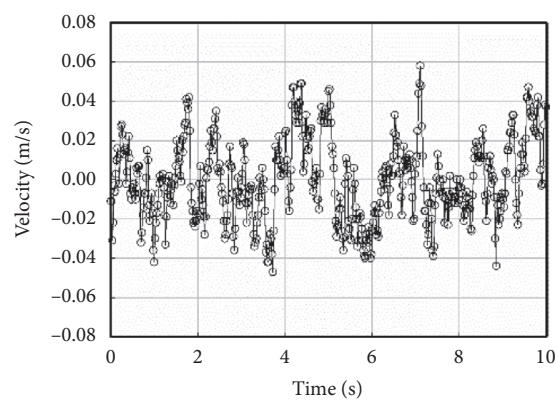

(h)

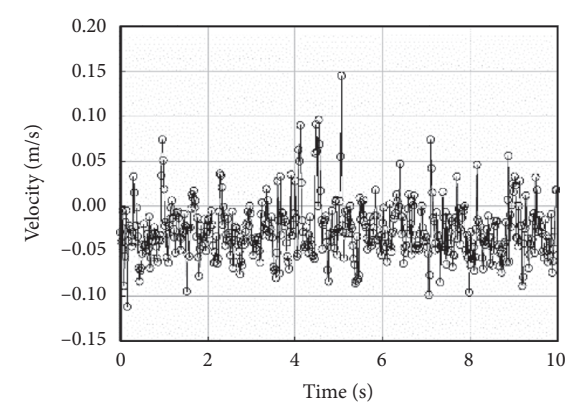

(c)

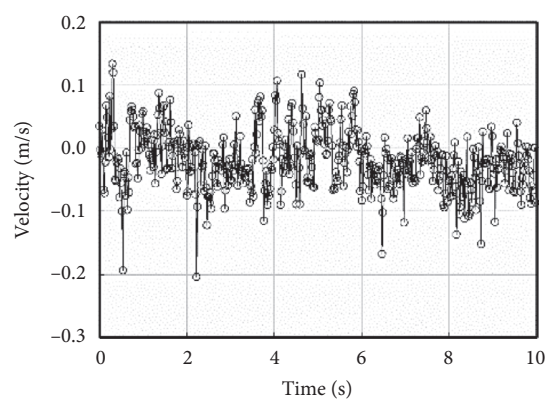

(f)

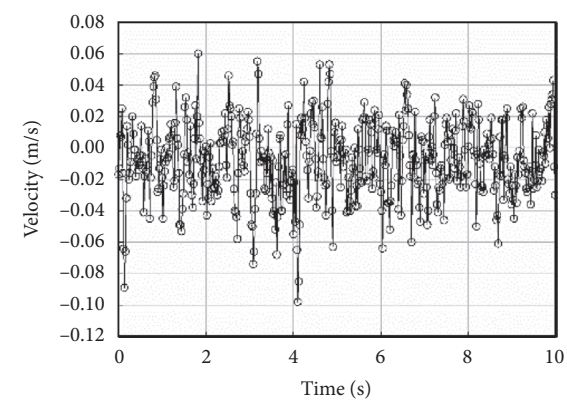

(i)

Figure 2: Instantaneous velocities $u, v$, and $w$ at sections 3, 7, and 11 in the experiments. (a) Velocity $u$ at section 3 . (b) Velocity $v$ at section 3. (c) Velocity $w$ at section 3. (d) Velocity $u$ at section 7. (e) Velocity $v$ at section 7. (f) Velocity $w$ at section 7. (g) Velocity $u$ at section 11. (h) Velocity $v$ at section 11 . (i) Velocity $w$ at section 11.

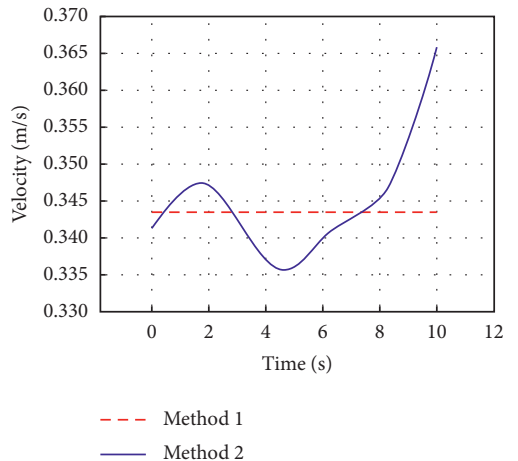

(a)

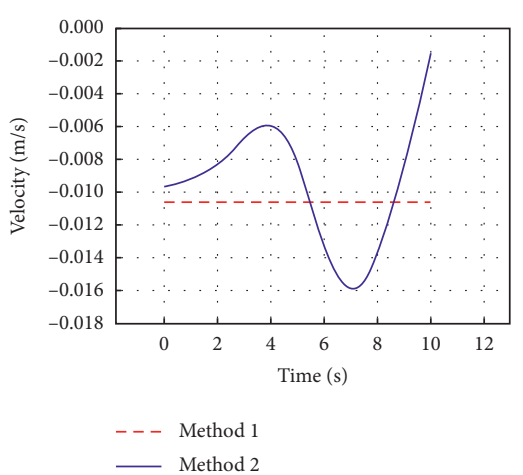

(b)

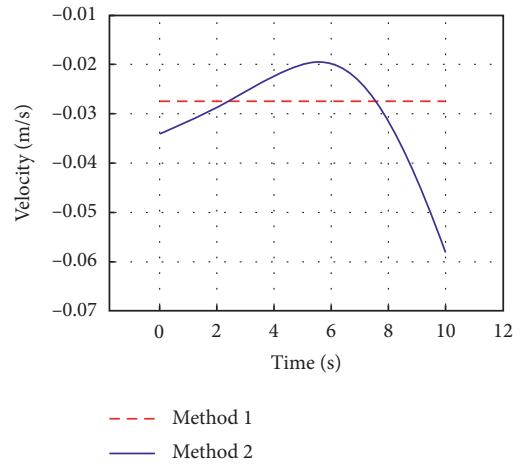

(c)

Figure 3: Continued. 


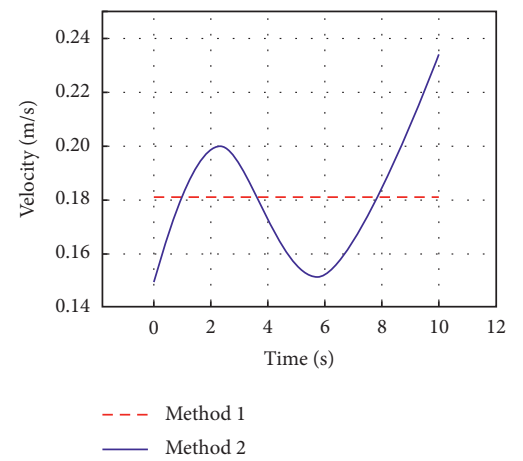

(d)

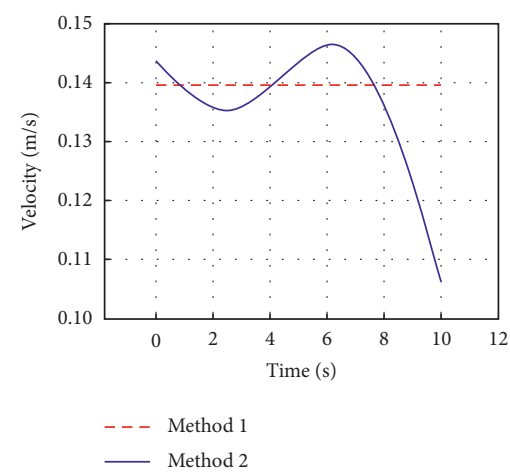

(g)

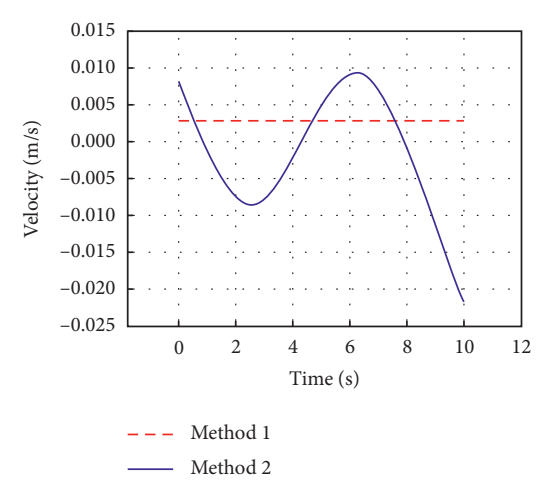

(e)

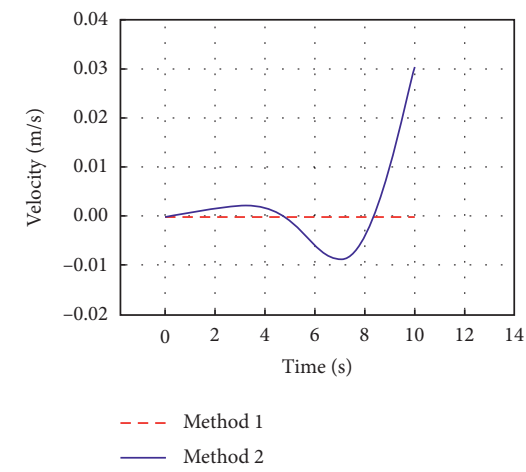

(h)

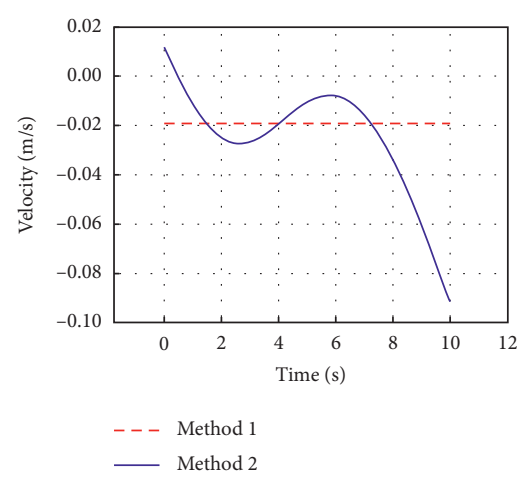

(f)

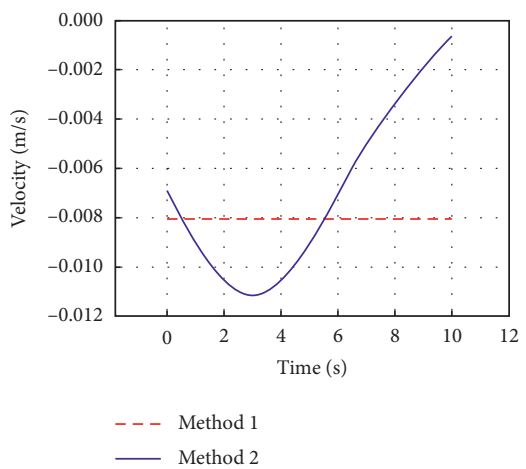

(i)

Figure 3: Mean velocities $u, v$, and $w$ at sections 3, 7, and 11 obtained using different methods. Methods 1 and 2 correspond to the mathematical expectation and adaptive global mean, respectively. (a) Mean velocity $u$ at section 3. (b) Mean velocity $v$ at section 3. (c) Mean velocity $w$ at section 3. (d) Mean velocity $u$ at section 7. (e) Mean velocity $v$ at section 7. (f) Mean velocity $w$ at section 7. (g) Mean velocity $u$ at section 11. (h) Mean velocity $v$ at section 11. (i) Mean velocity $w$ at section 11.

As shown in Figure 4, the pulsating velocities obtained using the two methods are consistent in terms of the velocity magnitude, fluctuation range, and fluctuation trend (Figures 4(a), 4(d), 4(g), 4(j), 4(m), 4(p), 4(s), 4(v), and 4(y)). This finding indicates that method 2 is an effective approach to calculate the pulsating velocity. Nevertheless, the pulsating velocities obtained using the two methods involve certain differences, which are more notable in the middle and later segments of the curves, especially in the later segments (Figures 4(c), 4(i), 4(k), 4(l), 4(o), 4(r), 4(u), and $4(\mathrm{x}))$. To further examine the differences in the data obtained using the two methods, the SPSS software was used to conduct a paired sample test (Table 1). As indicated in Table 1, except for pair 4 , the $P$ value for all the pairs was less than 0.05 , indicating that each pair of data involved significant differences.

3.4. Turbulent Characteristics. Turbulent characteristics are key features of flume turbulence. The relative turbulence intensity (TI), Reynolds shear stress $\left(\tau_{i j}\right)$, and turbulent kinetic energy (TKE) are the most commonly used indexes in practice $[15,17]$. The parameters can be calculated as follows:

$$
\begin{aligned}
\mathrm{TI}_{u} & =\frac{\sqrt{\left(u^{\prime}\right)^{2}}}{U_{0}}, \\
\mathrm{TI}_{v} & =\frac{\sqrt{\left(v^{\prime}\right)^{2}}}{U_{0}}, \\
\mathrm{TI}_{w} & =\frac{\sqrt{\left(w^{\prime}\right)^{2}}}{U_{0}}, \\
\tau_{i j} & =-\rho \overline{u_{i}^{\prime} u_{j}^{\prime}}, \\
\mathrm{TKE} & =\frac{\rho\left({\overline{u^{\prime}}}^{2}+{\overline{v^{\prime}}}^{2}+{\overline{w^{\prime}}}^{2}\right)}{2},
\end{aligned}
$$

where $\mathrm{TI}_{u}, \mathrm{TI}_{v}$, and $\mathrm{TI}_{w}$ denote the relative turbulence intensities in $u, v$, and $w$ directions, respectively; $u^{\prime}, v^{\prime}$, and $w^{\prime}$ denote the different components of the pulsating velocity; $U_{0}$ is the average velocity at a given section; and $\rho$ is the flow density. Figure 5 compares the turbulent characteristics determined using methods 1 and 2. 


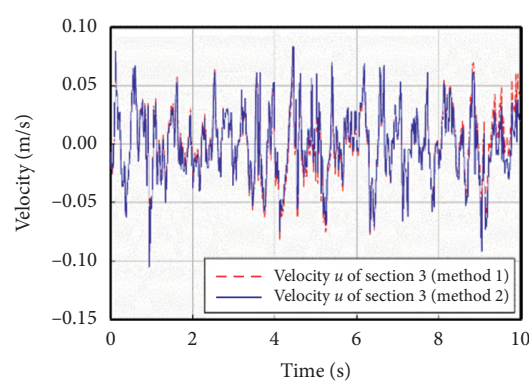

(a)

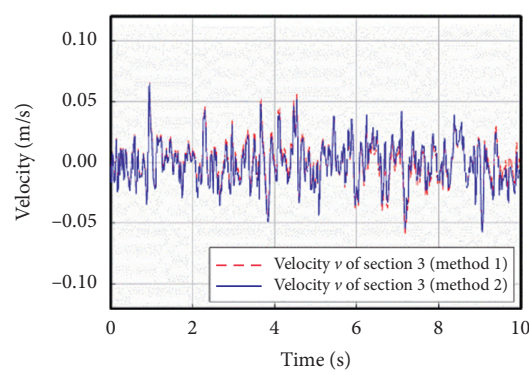

(d)

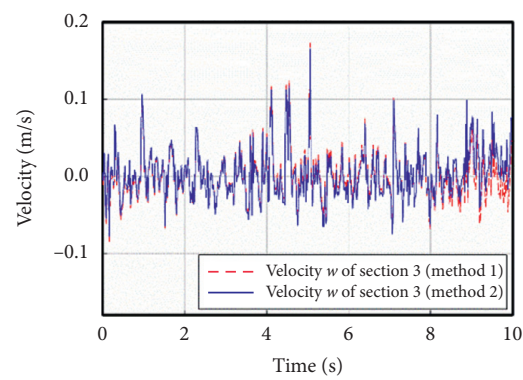

(g)

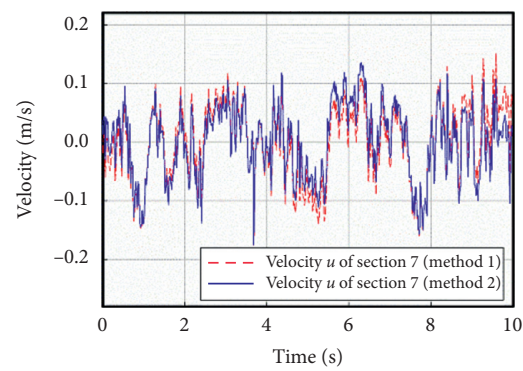

(j)

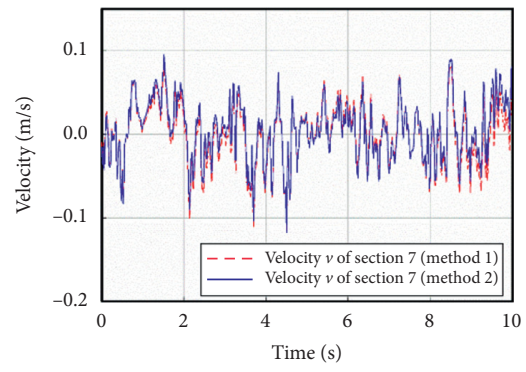

(m)

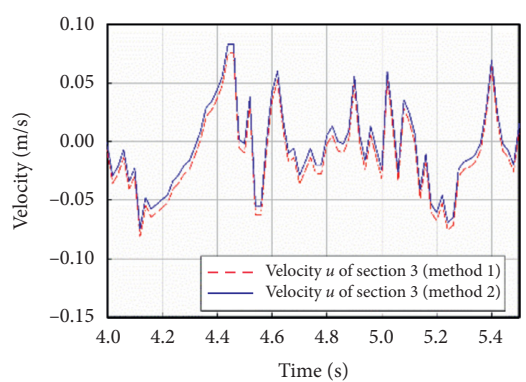

(b)

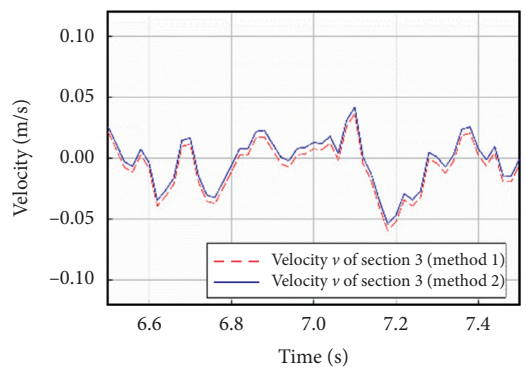

(e)

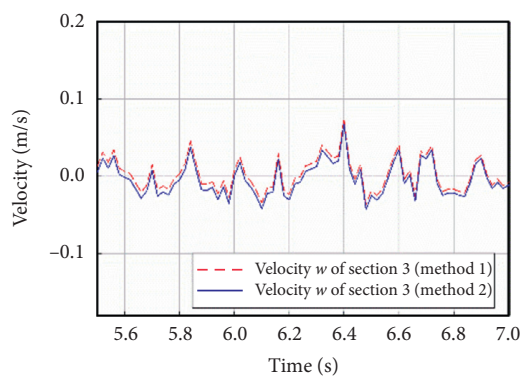

(h)

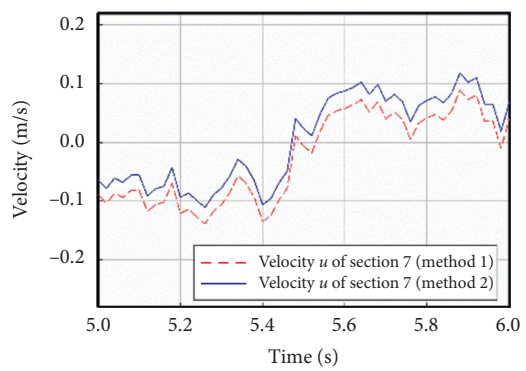

(k)

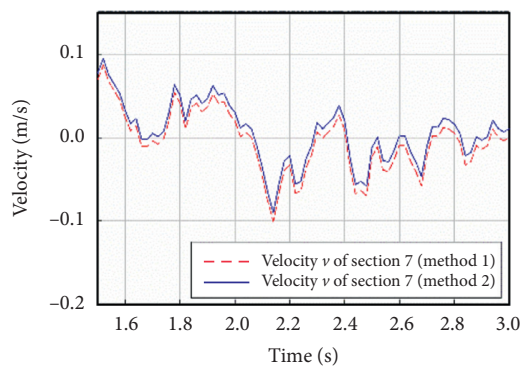

(n)

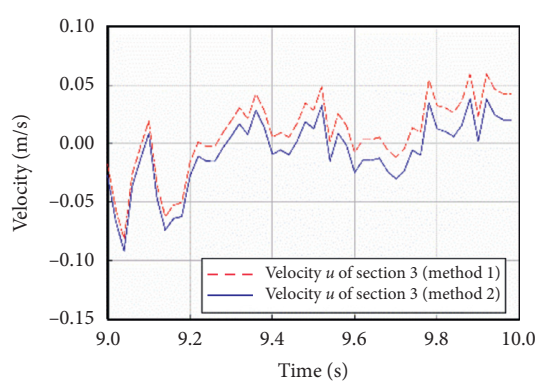

(c)

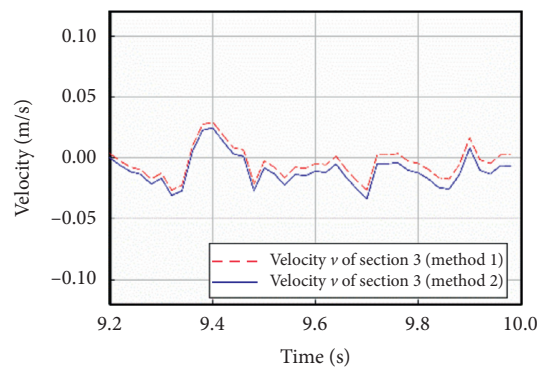

(f)

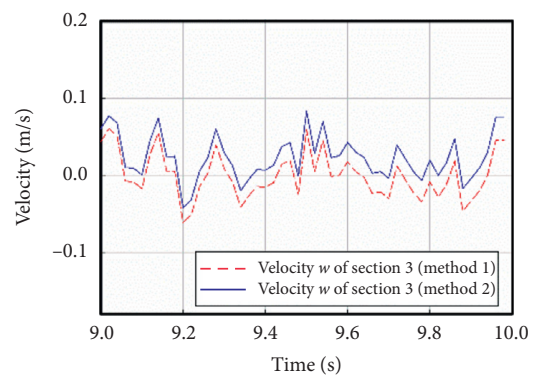

(i)

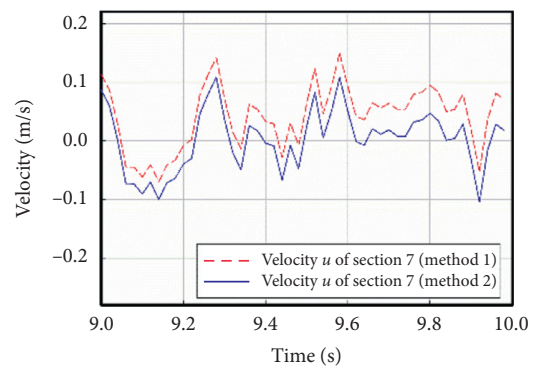

(1)

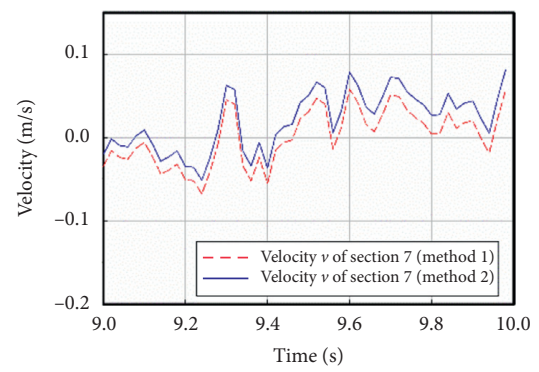

(o)

Figure 4: Continued. 


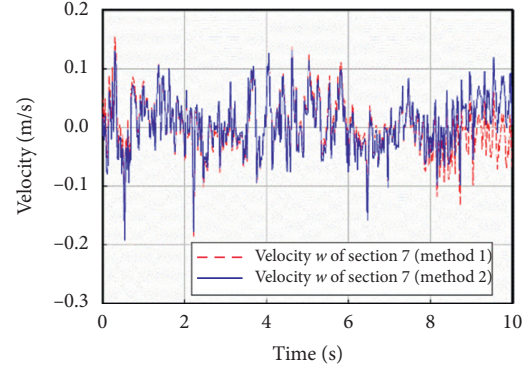

(p)

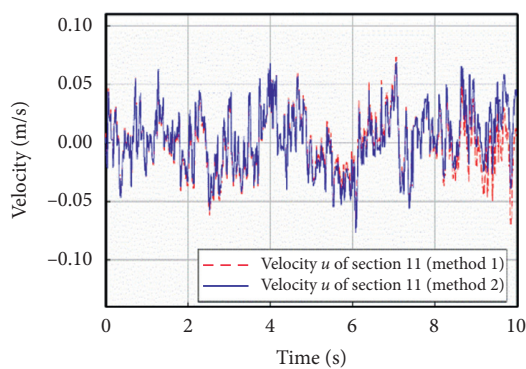

(s)

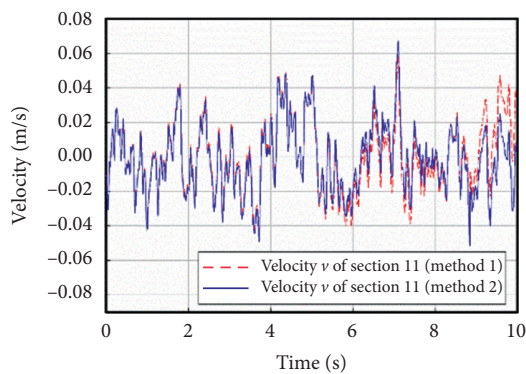

(v)

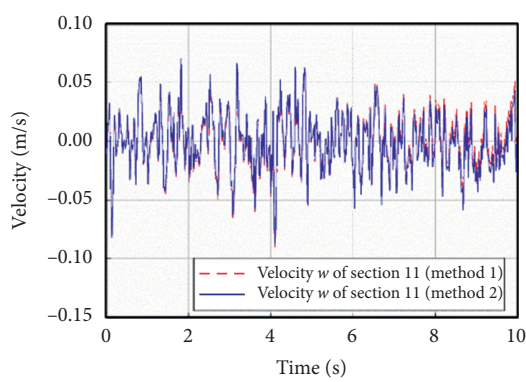

(y)

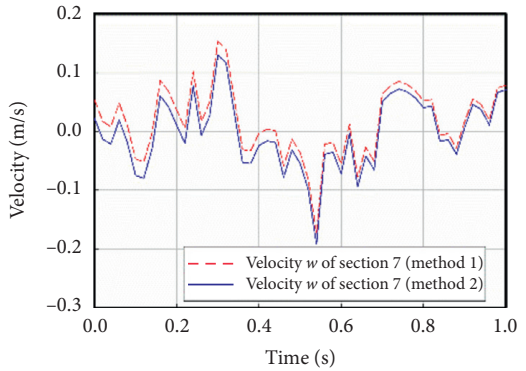

(q)

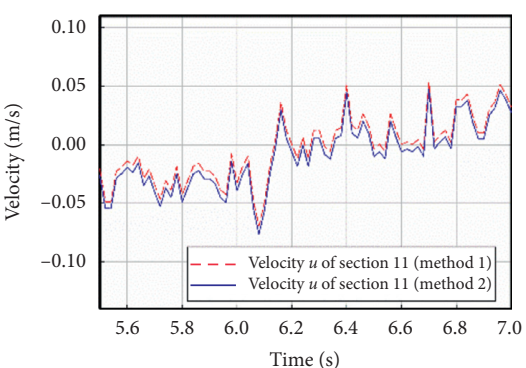

(t)

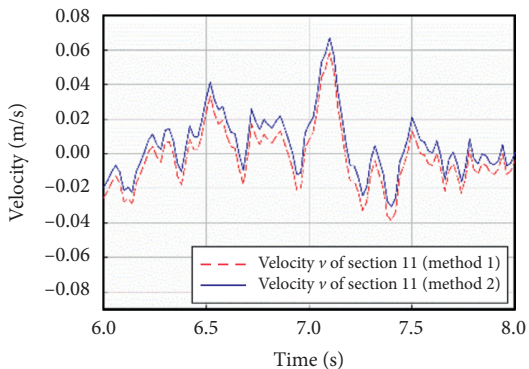

(w)

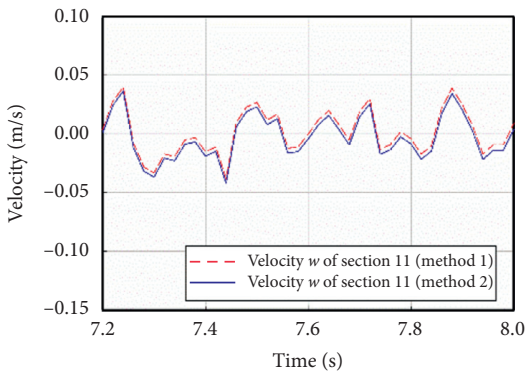

(z)

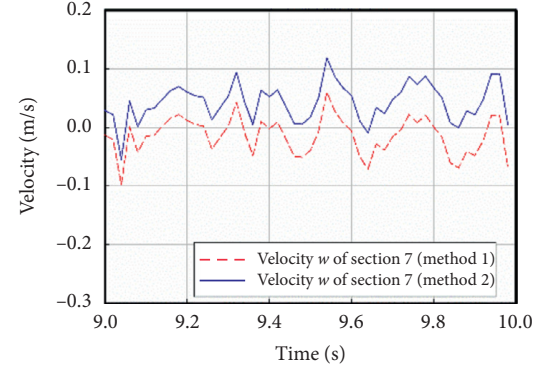

(r)

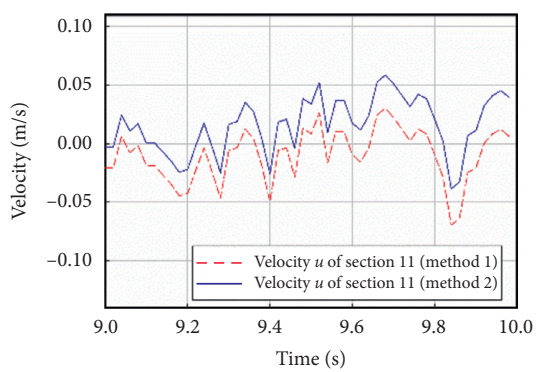

(u)

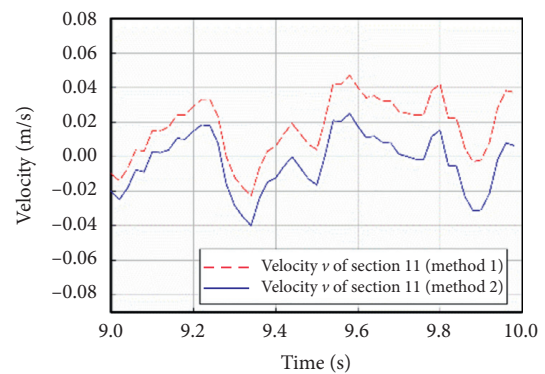

(x)

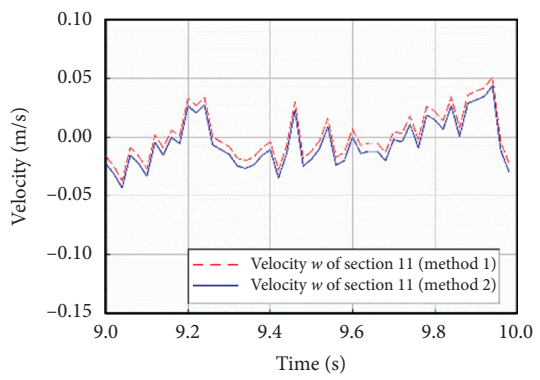

(a1)

Figure 4: Pulsating velocity $u, v$, and $w$ at sections 3, 7, and 11 obtained using different methods. Methods 1 and 2 correspond to the mathematical expectation and adaptive global mean, respectively.

As shown in Figure 5, the three turbulence characteristics calculated using the two methods are highly consistent in terms of magnitude and range. This finding validates that the ESMD method (method 2) is accurate and can be used to analyse the turbulence characteristics. Furthermore, the decomposed characteristics shown in the figure clearly highlight that (1) along the direction of the flow ( $u 3, u 7$, and $u 11$ ), the turbulence intensity does not change considerably; the Reynolds shear stress and the turbulent kinetic energy first increase and later decrease, with a considerable change in the turbulent kinetic energy. (2) Along the direction of the water depth $(v 3, v 7$, and $v 11)$, due to the flume shape, a drop flow occurs (section 3 ), and the water surface tends to rise after section 11. Subsequently, the turbulence intensity and Reynolds shear stress exhibit negative values. This finding indicates that a strong turbulence occurs at section 7 , in which all the three turbulence characteristics exhibit a large magnitude. Vowinckel et al. [18] also observed the same trend and indicated that in the case, the particles altered the flow field, thereby substantially enhancing the turbulence intensity and modifying the size of the coherent particle structure. (3) Along the direction of the flume width $(w 3, w 7$, and $w 11)$, the 
TABLE 1: Paired sample test results for the pulsating velocity.

\begin{tabular}{|c|c|c|c|c|c|c|c|c|c|}
\hline \multirow{3}{*}{ Pair $i$} & \multirow{3}{*}{$\begin{array}{l}\text { Pulsating velocity } \\
\text { (methods 1-2) }\end{array}$} & \multirow{3}{*}{ Mean } & \multicolumn{4}{|c|}{ Paired differences } & \multirow{3}{*}{$t$} & \multirow{3}{*}{$\mathrm{d} f$} & \multirow{3}{*}{ Significance (2-tailed) } \\
\hline & & & $\begin{array}{l}\text { Standard } \\
\text { deviation }\end{array}$ & $\begin{array}{c}\text { Standard error } \\
\text { mean }\end{array}$ & \multicolumn{2}{|c|}{$\begin{array}{l}95 \% \text { confidence } \\
\text { interval of the } \\
\text { difference }\end{array}$} & & & \\
\hline & & & & & Lower & Upper & & & \\
\hline Pair 1 & Velocity $u$ at section 3 & 0.00084 & 0.00648 & 0.00029 & 0.00027 & 0.00141 & 2.913 & 499 & 0.004 \\
\hline Pair 2 & Velocity $v$ at section 3 & 0.00100 & 0.00345 & 0.00015 & 0.00069 & 0.00130 & 6.457 & 499 & 0.000 \\
\hline Pair 3 & Velocity $w$ at section 3 & -0.00184 & 0.00923 & 0.00041 & -0.00266 & -0.00103 & -4.470 & 499 & 0.000 \\
\hline Pair 4 & Velocity $u$ at section 7 & -0.00086 & 0.02121 & 0.00095 & -0.00273 & 0.00100 & -0.912 & 499 & 0.362 \\
\hline Pair 5 & Velocity $v$ at section 7 & -0.00440 & 0.00775 & 0.00035 & -0.00508 & -0.00372 & -12.694 & 499 & 0.000 \\
\hline Pair 6 & Velocity $w$ at section 7 & -0.00592 & 0.02145 & 0.00096 & -0.00781 & -0.00404 & -6.173 & 499 & 0.000 \\
\hline Pair 7 & Velocity $u$ at section 11 & -0.00281 & 0.00903 & 0.00040 & -0.00361 & -0.00202 & -6.965 & 499 & 0.000 \\
\hline Pair 8 & Velocity $v$ at section 11 & 0.00107 & 0.00779 & 0.00035 & 0.00039 & 0.00175 & 3.074 & 499 & 0.002 \\
\hline Pair 9 & Velocity $w$ at section 11 & 0.00089 & 0.00336 & 0.00015 & 0.00059 & 0.00118 & 5.899 & 499 & 0.000 \\
\hline
\end{tabular}

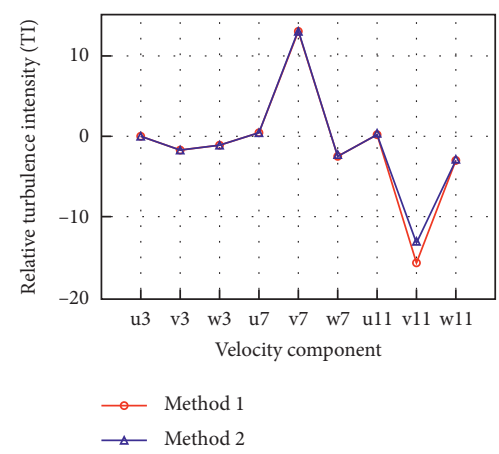

(a)

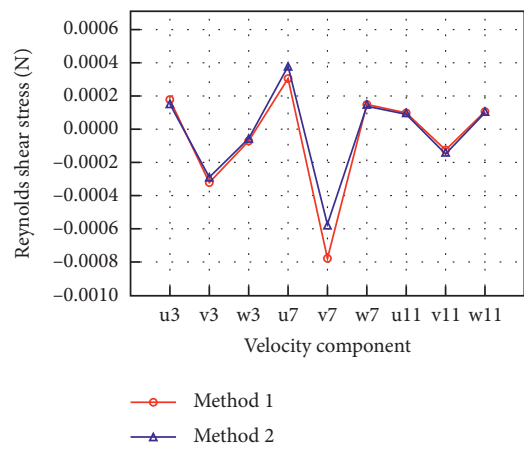

(b)

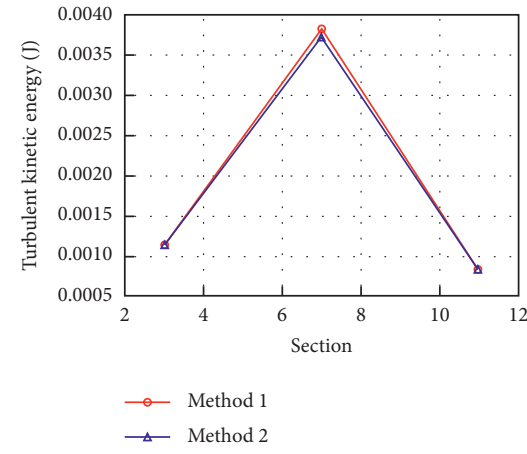

(c)

Figure 5: Turbulent characteristics of velocities $u, v$, and $w$ at sections 3, 7, and 11 obtained using different methods. Methods 1 and 2 correspond to the mathematical expectation and adaptive global mean, respectively. (a) Relative turbulence intensity. (b) Reynolds shear stress. (c) Turbulent kinetic energy.

turbulence intensity and Reynolds shear stress did not change considerably, thereby indicating that the flume width did not considerably influence the turbulence.

\section{Conclusion}

In this study, to analyse the feasibility of the ESMD method in determining the turbulence characteristics in flume flow, the traditional mathematical expectation method and ESMD method were used to analyse the turbulence characteristics of a solid-liquid flow in a flume. The results demonstrated that the method can be efficiently used to perform the turbulence analysis of two-phase flows in a flume. The following conclusions were derived from the results:

(1) In the context of the traditional mathematical expectation approach to calculate the pulsating velocity, the ESMD method is a new and powerful alternative method to perform the turbulent analysis of solid-liquid flows. The theoretical basis of the ESMD method is correct, and the obtained results are more consistent with the turbulence behaviour in the flume.

(2) The optimal adaptive global mean (AGM), which is the result of modal decomposition, can suitably reflect the overall tendency of the instantaneous velocity, along with the turbulence response at different times

(3) The turbulence characteristics calculated based on the ESMD method can effectively reflect the turbulence behaviour in the flume which is important to further understand and predict the flow pattern, the recirculation zone or the scour area, and so on.

\section{Nomenclature}

$d_{50}: \quad$ Median diameter of a solid particle

$f^{*}(t): \quad$ Instantaneous frequency

$\bar{V}: \quad$ Mean velocity

$v_{i}: \quad$ Instantaneous velocity

$N$ : Number of points for which the instantaneous velocity is considered

$\mathrm{TI}_{u}$ : $\quad$ Relative turbulence intensity in the $u$ direction

$\mathrm{TI}_{v}$ : $\quad$ Relative turbulence intensity in the $v$ direction

$\mathrm{TI}_{w}$ : $\quad$ Relative turbulence intensity in the $w$ direction $u^{\prime}, v^{\prime}, w^{\prime}$ : Different components of the pulsating velocity

$U_{0}: \quad$ Average velocity for a given section

$\rho: \quad$ Flow density

$\tau_{i j}$ : $\quad$ Reynolds shear stress

TKE: Turbulent kinetic energy. 


\section{Data Availability}

The data of Figures 2-5 used to support the findings of this study are available from the Jiangsu Vocational Institute of Architectural Technology (szp821002@126.com).

\section{Conflicts of Interest}

The authors declare that they have no conflicts of interest.

\section{Acknowledgments}

This study benefited from the valuable and critical suggestions provided by the referees and the editor. This work was supported by the National Natural Science Foundation of China (51879227), Science and Technology Project of Department of Housing and Urban-Rural Development of Jiangsu Province (2019ZD082), Qinglan Project in the University of Jiangsu Province (2021), and Natural Science Project of Jiangsu Vocational Institute of Architectural Technology (JYJBZX19-05).

\section{References}

[1] M. Fettweis, M. Baeye, F. Francken et al., "Monitoring the effects of disposal of fine sediments from maintenance dredging on suspended particulate matter concentration in the Belgian nearshore area (Southern North Sea)," Marine Pollution Bulletin, vol. 62, no. 2, pp. 258-269, 2011.

[2] G. Wang, S. Zhou, J. B. Joshi, G. J. Jameson, and G. M. Evans, "An energy model on particle detachment in the turbulent field," Minerals Engineering, vol. 69, pp. 165-169, 2014.

[3] J. Chen, H. Li, X. Lv, and Q. Zhu, "A structure-based drag model for the simulation of Geldart A and B particles in turbulent fluidized beds," Powder Technology, vol. 274, pp. 112-122, 2015.

[4] K. Sun, L. Lu, H. Jiang, and H. H. Jin, "Experimental study of solid particle deposition in $90^{\circ}$ ventilated bends of rectangular cross section with turbulent flow," Aerosol Science and Technology, vol. 47, no. 2, pp. 115-124, 2012.

[5] X. Liu and M. ASCE, "New near-wall treatment for suspended sediment transport simulations with high Reynolds number turbulence models," Journal of Hydraulic Engineering, vol. 140, no. 3, pp. 333-339, 2014.

[6] N. S. Chen, "Vegetation effects on open channel flows and sediment transport," Journal of Hydroelectric Engineering, vol. 37, no. 9, pp. 1-7, 2018, in Chinese.

[7] L. Zhong, T. Jiang, J. M. Zhang, and J. J. Liu, "PIV experimental study on velocity fluctuations of turbulent flow in open channel," Advanced Engineering Sciences, vol. 51, no. 4, pp. 84-93, 2019, in Chinese.

[8] C. Venier, J. Figueiredo da Silva, S. J. Mclelland, R. W. Duck, and S. Lanzoni, "Experimental investigation of the impact of macroalgal mats on flow dynamics and sediment stability in shallow tidal areas," Estuarine, Coastal and Shelf Science, vol. 112, pp. 52-60, 2012.

[9] X. Liu, Y. Tang, Z. Lu, H. Huang, X. Tong, and J. Ma, "ESMDbased stability analysis in the progressive collapse of a building model: a case study of a reinforced concrete frame-shear wall model," Measurement, vol. 120, pp. 34-42, 2018.

[10] J. L. Wang and Z. J. Li, "Extreme-point symmetric mode decomposition method for data analysis," Advance in Adaptive Data Analysis, vol. 5, no. 3, Article ID 1350015, 2013.
[11] Y. Qin, B. Li, Z. Chen, Y. Chen, and L. Lian, "Spatio-temporal variations of nonlinear trends of precipitation over an arid region of northwest China according to the extreme-point symmetric mode decomposition method," International Journal of Climatology, vol. 38, no. 5, pp. 2239-2249, 2017.

[12] F. Wang, Z. Wang, H. Yang, and Y. Zhao, "Study of the temporal and spatial patterns of drought in the Yellow River basin based on SPEI," Science China Earth Sciences, vol. 61, no. 8, pp. 1098-1111, 2018.

[13] Y. Ren, H. Zhang, W. Wei, X. Cai, Y. Song, and L. Kang, “A study on atmospheric turbulence structure and intermittency during heavy haze pollution in the Beijing area," Science China Earth Sciences, vol. 62, no. 12, pp. 2058-2068, 2019, in Chinese.

[14] K. M. O. Håkansson, M. Kvick, F. Lundell, L. Prahl Wittberg, and L. D. Söderberg, "Measurement of width and intensity of particle streaks in turbulent flows," Experiments in Fluids, vol. 54, no. 6, p. 1555, 2013.

[15] E. Izadinia, M. Heidarpour, and A. J. Schleiss, "Investigation of turbulence flow and sediment entrainment around a bridge pier," Stochastic Environmental Research and Risk Assessment, vol. 27, no. 6, pp. 1303-1314, 2013.

[16] D. Cava, A. Donateo, and D. Contini, "Combined stationarity index for the estimation of turbulent fluxes of scalars and particles in the atmospheric surface layer," Agricultural and Forest Meteorology, vol. 194, pp. 88-103, 2014.

[17] M. Bizhani, F. E. R. Corredor, and E. Kuru, "An experimental study of turbulent non-Newtonian Fluid flow in concentric Annuli using particle image velocimetry technique," Flow, Turbulence and Combustion, vol. 94, no. 3, pp. 527-554, 2015.

[18] B. Vowinckel, T. Kempe, and J. Fröhlich, "Fluid-particle interaction in turbulent open channel flow with fully-resolved mobile beds," Advances in Water Resources, vol. 72, pp. 32-44, 2014. 